\title{
Convergence Properties of the Membership Set in the Presence of Disturbance and Parameter Uncertainty ${ }^{\dagger}$
}

\author{
Wataru KITAMURA* and Yasumasa FujISAKi**
}

\begin{abstract}
The size of the membership set is investigated in the presence of bounded disturbance and $l_{2}$ bounded parameter uncertainty. A tight upper bound of the diameter of the membership set is derived in a deterministic setting, which indicates that the diameter does not converge to zero in general. Then, a probabilistic upper bound of the diameter is derived in a stochastic setting, where the disturbance and the parameter uncertainty are assumed to be random variables and to take a value near the worst ones with a probability. This probabilistic bound leads to the fact that the diameter converges to zero with probability one as the number of samples tends to infinity.
\end{abstract}

Key Words: Set membership identification, uncertainty, diameter, convergence, stochastic setting.

\section{Introduction}

The membership set represents the entire set of system parameters that are consistent with measured data and a given bound of disturbance, and it is useful for robustness analysis of system identification. Nowadays, many algorithms of set membership identification are available ${ }^{1) \sim 5 \text { ), }}$ which produce the membership set itself or an optimal parameter in it. On the other hand, there are a few studies focused on fundamental properties of the membership set ${ }^{6) ~ 9)}$. In the studies, except for the authors' previous work ${ }^{9)}$, the properties have been explored for systems with additive bounded disturbance but no uncertainty in the system parameter.

In this paper, we concentrate our attention on a fundamental property, that is, convergence property of the membership set with respect to the number of samples. We investigate the membership set in the presence of not only disturbance but also parameter uncertainty as the authors' previous work ${ }^{9)}$. However, instead of an $l_{1}$ bounded uncertainty ${ }^{9}$, we here consider an $l_{2}$ bounded uncertainty. Since the $l_{2}$ norm is more compatible with persistently exciting condition than the $l_{1}$ norm, we can expect to derive a simple formula that is easy to understand, which is demonstrated in this paper.

We first introduce a system with an additive bounded

+ The preliminary version of this paper was presented in the 41 st SICE Annual Conference $(2002 \cdot 8)$

* Graduate School of Science and Technology, Kobe University, Kobe

** Department of Computer and Systems Engneering, Kobe University, Kobe

(Received July 8, 2002)

(Revised December 9, 2002) disturbance and an $l_{2}$ bounded parameter uncertainty, and define the membership set. Then, we derive a characterization of the membership set, which has a convenient formula. Based on this characterization, a tight upper bound of.the diameter of the membership set is directly obtained in a deterministic setting, which indicates that the diameter does not converge to zero in general. This motivates us to provide another upper bound of the diameter in a stochastic setting ${ }^{7) ~ 9)}$, where we assume that the disturbance and the parameter uncertainty are random variables and their bounds are tight, that is, they take a value near the worst ones with a probability. The obtained probabilistic upper bound of the diameter indicates that the diameter converges to zero with probability one if the bounds of the disturbance and the parameter uncertainty are tight and the regressor is deterministic and persistently exciting. The result also indicates the necessary number of samples which guarantees that the resultant diameter of the membership set is less than a given bound with a given probabilistic confidence: $\mathrm{No}-$ tice that there are some researches on finite sample analysis ${ }^{10) ~ 12)}$, but they have not investigated convergence property of the membership set. A numerical example illustrates the obtained result.

\section{The Membership Set}

Let us describe a single-input single-output (SISO) discrete time system as

$$
y_{i}=\phi_{i}^{\mathrm{T}}\left(\theta+\eta_{i}\right)+v_{i}
$$

where $y_{i} \in \mathbf{R}$ is the system output, $\phi_{i} \in \mathbf{R}^{\mathbf{m}}$ is the measurable regressor vector, $\theta \in \mathbf{R}^{\mathbf{m}}$ is the true but unknown parameter to be identified, $v_{i} \in \mathbf{R}$ is an unknown additive disturbance, and $\eta_{i} \in \mathbf{R}^{\mathbf{m}}$ is an unknown parameter 
uncertainty at time $i=1,2, \ldots, N$. The disturbance and parameter uncertainty are bounded, that is,

$$
\left|v_{i}\right| \leq \epsilon, \quad\left\|\eta_{i}\right\|_{2} \leq \delta
$$

where the bounds $\epsilon$ and $\delta$ are given, and \|\|$_{2}$ is the $l_{2}$ norm

$$
\left\|\eta_{i}\right\|_{2} \doteq\left(\eta_{i}^{\mathbf{T}} \eta_{i}\right)^{1 / 2}
$$

Then, the membership set is defined as all estimates $\hat{\theta}$ that are consistent with the given date and the given bounds of the disturbance and the parameter uncertainty, that is,

$$
\begin{aligned}
& \Omega_{0}^{N}(\epsilon, \delta) \doteq \bigcap_{i=1}^{N}\left\{\hat{\theta} \in \mathbf{R}^{m}: \exists v_{i}, \exists \eta_{i}\right. \\
& \text { s.t. } \left.y_{i}=\phi_{i}^{\mathrm{T}}\left(\hat{\theta}+\eta_{i}\right)+v_{i},\left|v_{i}\right| \leq \epsilon,\left\|\eta_{i}\right\|_{2} \leq \delta\right\} .
\end{aligned}
$$

To obtain a convenient representation of the membership set, we first rewrite the system (1) as

$$
y_{i}-\phi_{i}^{\mathbf{T}} \theta=v_{i}+\phi_{i}^{\mathbf{T}} \eta_{i}
$$

where the constraints (2) imply

$$
\left|v_{i}+\phi_{i}^{\mathrm{T}} \eta_{i}\right| \leq \epsilon+\delta\left\|\phi_{i}\right\|_{2}
$$

Notice that the equality in the above can be attained with

$$
v_{i}= \pm \epsilon, \quad \eta_{i}= \pm \frac{\delta}{\left\|\phi_{i}\right\|_{2}} \phi_{i}
$$

which meet (2). Thus, we obtain another characterization of the membership set:

$$
\begin{aligned}
& \Omega_{1}^{N}(\epsilon, \delta) \doteq \bigcap_{i=1}^{N}\left\{\hat{\theta} \in \mathbf{R}^{m}:\right. \\
& \left.\quad-\left(\epsilon+\delta\left\|\phi_{i}\right\|_{2}\right) \leq y_{i}-\phi_{i}^{\mathbf{T}} \hat{\theta} \leq \epsilon+\delta\left\|\phi_{i}\right\|_{2}\right\} .
\end{aligned}
$$

Note that $\Omega_{0}^{N}(\epsilon, \delta)=\Omega_{1}^{N}(\epsilon, \delta)$ and the quantifiers in the definition of $\Omega_{0}^{N}(\epsilon, \delta)$ are eliminated in the characterization $\Omega_{1}^{N}(\epsilon, \delta)$. In the rest of this paper, we use this convenient characterization $\Omega_{1}^{N}(\epsilon, \delta)$.

We remark that the parameter uncertainty behaves as an additional disturbance and the resultant bound $\epsilon+\delta\left\|\phi_{i}\right\|_{2}$ depends on the regressor and is time varying. Hence, to identify this membership set, we may use the existing algorithms with some modifications.

\section{The Size of the Membership Set}

Let us define the diameter of the membership set as

$$
\operatorname{dia} \Omega_{1}^{N}(\epsilon, \delta) \doteq \sup _{\hat{\theta}_{1}, \hat{\theta}_{2} \in \Omega_{1}^{N}(\epsilon, \delta)}\left\|\hat{\theta}_{1}-\hat{\theta}_{2}\right\|_{2} .
$$

The diameter is an index of the size of the membership set. In this section, we take a deterministic setting, that is, we derive the diameter based only on the bounds $\epsilon$ and $\delta$. On the other hand, we will take a stochastic setting in the following sections, where $v_{i}$ and $\eta_{i}$ are assumed to be random variables.
The following is a standard definition of persistently exciting ${ }^{7), 8)}$ in set membership identification.

Definition 1. The regressor $\phi_{i}$ is said to be persistently exciting (PE) if there exist some $\alpha, \beta$, and $n_{0} \geq m$ such that

$$
\alpha^{2} I \leq \sum_{i=i_{i}}^{i_{0}+n_{11}-1} \phi_{i} \phi_{i}^{T} \leq \beta^{2} I
$$

for all $i_{0} \geq 1$. Here, the matrix inequality $A \leq B$ means $x^{T}(B-A) x \geq 0$ for all $x$.

Note that both deterministic and stochastic regressors can be PE.

The PE condition ensures the boundedness of the membership set, and is assumed throughout this paper. For simplicity, let $N=\ell n_{0}$, where $\ell$ is an integer. Then, we obtain the result.

Theorem 1. Consider the system (1). Assume that the regressor $\phi_{i}$ is deterministic and PE. Then,

$$
\operatorname{dia} \Omega_{1}^{N}(\epsilon, \delta) \leq \frac{2 \sqrt{n_{0}}}{\alpha}(\epsilon+\delta \beta) .
$$

Proof: Rewrite the given data as a compact form

$$
y \doteq\left[\begin{array}{c}
y_{1} \\
y_{2} \\
\vdots \\
y_{N}
\end{array}\right] \in \mathbf{R}^{N}, \quad \Phi^{N} \doteq\left[\begin{array}{c}
\phi_{1}^{\mathrm{T}} \\
\phi_{2}^{\mathbf{T}} \\
\vdots \\
\phi_{N}^{\mathrm{T}}
\end{array}\right] \in \mathbf{R}^{N \times m}
$$

The PE condition implies

$$
\left(\Phi^{N}\right)^{\mathrm{T}} \Phi^{N}=\sum_{i=1}^{\ell n_{0}} \phi_{i} \phi_{i}^{\mathrm{T}} \geq \alpha^{2} \ell I
$$

thus we have

$$
\left\|\left\{\left(\Phi^{N}\right)^{\mathbf{T}} \Phi^{N}\right\}^{-1}\left(\Phi^{N}\right)^{\mathrm{T}}\right\|_{i 2} \leq \frac{1}{\alpha \sqrt{\ell}}
$$

where $\|\bullet\|_{i 2}$ is the $l_{2}$ induced norm, that is,

$$
\|A\|_{i 2} \doteq \sup _{x \neq 0} \frac{\|A x\|_{2}}{\|x\|_{2}}=\sqrt{\bar{\lambda}\left(A A^{\mathrm{T}}\right)}
$$

where $A$ is a real matrix and $\bar{\lambda}\left(A A^{\mathrm{T}}\right)$ is the largest eigenvalue of $A A^{\mathrm{T}}$. Furthermore, for any $i$, there exists $i_{\mathrm{p}}$ such that $i_{0} \leq i \leq i_{0}+n_{0}-1$. Then, the PE condition implies

$$
\phi_{i} \phi_{i}^{\mathrm{T}} \leq \sum_{\hat{i}=i_{i}}^{i_{0}+n_{0}-1} \phi_{\hat{i}} \phi_{\hat{i}}^{\mathrm{T}} \leq \beta^{2} I
$$

thus

$$
\left\|\phi_{i}\right\|_{2} \leq \beta
$$

for all $i$. Hence, if $\hat{\theta}_{1}$ and $\hat{\theta}_{2}$ are in $\Omega_{1}^{N}(\epsilon, \delta)$, then

$$
\begin{aligned}
& -(\epsilon+\delta \beta) \leq y_{i}-\phi_{i}^{\mathrm{T}} \hat{\theta}_{1} \leq \epsilon+\delta \dot{\beta} \\
& -(\epsilon+\delta \beta) \leq y_{i}-\phi_{i}^{\mathrm{T}} \hat{\theta}_{2} \leq \epsilon+\delta \dot{\beta} .
\end{aligned}
$$

This implies

$$
-2(\epsilon+\delta \beta) \leq \phi_{i}^{\mathrm{T}}\left(\hat{\theta}_{1}-\hat{\theta}_{2}\right) \leq 2(\epsilon+\delta \beta)
$$


thus we have

$$
\left\|\Phi^{N}\left(\hat{\theta}_{1}-\hat{\theta}_{2}\right)\right\|_{2} \leq 2 \sqrt{N}(\epsilon+\ddot{\delta} \beta) .
$$

Using the inequalities (7) and (8), we obtain

$$
\begin{aligned}
\left\|\hat{\theta}_{1}-\hat{\theta}_{2}\right\|_{2} \leq & \left\|\left\{\left(\Phi^{N}\right)^{\mathrm{T}} \Phi^{N}\right\}^{-1}\left(\Phi^{N}\right)^{\mathrm{T}}\right\|_{i 2} \\
& \cdot\left\|\Phi^{N}\left(\hat{\theta}_{1}-\hat{\theta}_{2}\right)\right\|_{2} \\
\leq & \frac{2 \sqrt{N}}{\alpha \sqrt{\ell}}(\epsilon+\delta \beta) \\
= & \frac{2 \sqrt{n_{0}}}{\alpha}(\epsilon+\delta \beta)
\end{aligned}
$$

for all $\hat{\theta}_{1}, \hat{\theta}_{2} \in \Omega_{1}^{N}(\epsilon, \delta)$. We therefore conclude the proof.

We remark that this theorem includes the existing result $^{7)}$ as a special case. In fact, if there is no parameter uncertainty $(\delta=0)$, then the theorem reduces to the previous result.

This theorem gives only an upper bound of the diameter of the membership set. Thus the bound may be conservative. However, it should be noted that the bound is actually the diameter if $\delta=0^{7)}$ or if $\alpha=\beta$ and $n_{0}=m$ (in Appendix A). In this sense, the bound is not conservative. We therefore see that the size of the membership set does not necessarily decrease whenever the number of the samples increases.

\section{Convergence Property of the Mem- bership Set}

Now, we investigate convergence property of the membership set in a stochastic setting ${ }^{9)}$, where the disturbance and the parameter uncertainty are regarded as random variables.

The following definition is introduced in the reference ${ }^{7)}$, which is a probabilistic assumption on the disturbance.

Definition 2. Suppose that the disturbance $v_{i}$ is a random variable satisfying $\left|v_{i}\right| \leq \epsilon$. The bound $\epsilon$ is said to be tight if for any $\rho>0$ and each $i$

$$
\begin{aligned}
& \operatorname{Prob}\left\{-\epsilon \leq v_{i} \leq-(\epsilon-\rho)\right\} \geq p_{v}(\rho) \\
& \operatorname{Prob}\left\{\epsilon-\rho \leq v_{i} \leq \epsilon\right\} \geq p_{v}(\rho)
\end{aligned}
$$

for some $1 \geq p_{v}(\rho)>0$.

In this paper, we also introduce a probabilistic assumption on the parameter uncertainty.

Definition 3. Suppose that the parameter uncertainty $\eta_{i}$ is a random vector satisfying $\left\|\eta_{i}\right\|_{2} \leq \delta$. The bound $\delta$ is said to be tight if for any $\mu>0$, each $i$, and any $\phi_{i}$

$$
\begin{aligned}
& \operatorname{Prob}\left\{-\delta\left\|\phi_{i}\right\|_{2} \leq \phi_{i}^{\mathbf{T}} \eta_{i} \leq-(\delta-\mu)\left\|\phi_{i}\right\|_{2}\right\} \geq p_{\eta}(\mu) \\
& \operatorname{Prob}\left\{(\delta-\mu)\left\|\phi_{i}\right\|_{2} \leq \phi_{i}^{\mathrm{T}} \eta_{i} \leq \delta\left\|\phi_{i}\right\|_{2}\right\} \geq p_{\eta}(\mu)
\end{aligned}
$$

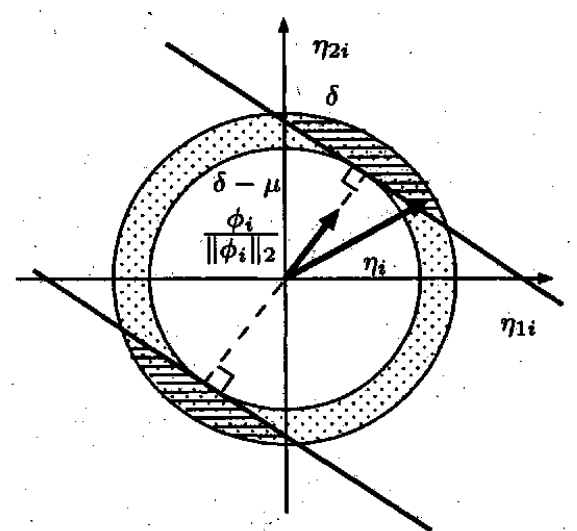

Fig. 1 Tightness in the case $m=2$

for some $1 \geq p_{\eta}(\mu)>0$.

The tightriess of the bounds of $v_{i}$ and $\eta_{i}$ means that $v_{i}$ and $\eta_{i}$ take a value around the surface of the possible values with nonzero probability. This is obvious for $v_{i}$, and can be seen for $\eta_{i}$ if we rewrite the formulae in Definition 3 as

$$
\begin{aligned}
& \operatorname{Prob}\left\{-\delta \leq \frac{\phi_{i}^{\mathbf{T}}}{\left\|\phi_{i}\right\|_{2}} \eta_{i} \leq-(\delta-\mu)\right\} \geq p_{\eta}(\mu) \\
& \operatorname{Prob}\left\{\delta-\mu \leq \frac{\phi_{i}^{\mathbf{T}}}{\left\|\phi_{i}\right\|_{2}} \eta_{i} \leq \delta\right\} \geq p_{\eta}(\mu) .
\end{aligned}
$$

Actually, when $m=2$, we can easily illustrate this probabilistic statement with Fig. 1. Suppose that the bound $\delta$ is tight with a parameter $\mu$. Then, for a fixed $\phi_{i}, \eta_{i}$ takes a value in each shaded portion with probability $p_{\eta}(\mu)$. Since this holds for any $\phi_{i}$, we see that, with a probability, $\eta_{i}$ takes a value in the point patterned portion, that is, around the surface of the possible values.

Notice that the definition is quite different from that in the previous paper ${ }^{9}$, where the parameter uncertainty is assumed to be bounded with $l_{1}$ norm and their extreme points are especially used in the definition of the tightness. On the other hand, in Definition 3, the surface of the parameter uncertainty are picked up in the entire set of the uncertainty, that is, all directions of the uncertainty are uniformly important.

The next lemma can be obtained immediately.

Lemma 1. Assume that the disturbance $v_{i}$ and the parameter uncertainty $\eta_{i}$ are independent random variables, and their bounds $\epsilon$ and $\delta$ are tight. Then,

$$
\begin{aligned}
& \operatorname{Prob}\left\{-\left(\epsilon+\delta\left\|\phi_{i}\right\|_{2}\right) \leq v_{i}+\phi_{i}^{\mathrm{T}} \eta_{i}\right. \\
& \left.\quad \leq-(\epsilon-\rho)-(\delta-\mu)\left\|\phi_{i}\right\|_{2}\right\} \geq p_{v}(\rho) p_{\eta}(\mu)>0 \\
& \operatorname{Prob}\left\{\epsilon-\rho+(\delta-\mu)\left\|\phi_{i}\right\|_{2} \leq v_{i}+\phi_{i}^{\mathrm{T}} \eta_{i}\right. \\
& \left.\quad \leq \epsilon+\delta\left\|\phi_{i}\right\|_{2}\right\} \geq p_{v}(\rho) p_{\eta}(\mu)>0 .
\end{aligned}
$$

That is, the bound $\epsilon+\delta\left\|\phi_{i}\right\|_{2}$ of $v_{i}+\phi_{i}^{\mathrm{T}} \eta_{i}$ is also tight. 
For the sake of simplicity, suppose that the regressor $\phi_{i}$ in (1) is periodic with period $n(n \geq m)$ and $N=\ell n$. Then, we can write

$$
\phi_{i_{p}+k n}=\phi_{i_{p}}
$$

where $i_{p}=1,2, \ldots, n, k=0,1,2, \ldots, \ell-1$, and $\ell \geq 1$. If the regressor is $\mathrm{PE}$, we can assume that $n_{0}=n$ in (5) without loss of generality, that is,

$$
\alpha^{2} I \leq\left(\Phi^{n}\right)^{\mathrm{T}} \Phi^{n} \leq \beta^{2} I
$$

holds for some $\alpha$ and $\beta$, where

$$
\Phi^{n} \doteq\left[\begin{array}{c}
\phi_{1}^{\mathrm{T}} \\
\phi_{2}^{\mathrm{T}} \\
\vdots \\
\phi_{n}^{\mathrm{T}}
\end{array}\right] \in \mathbf{R}^{n \times m}
$$

The following theorem is the main result of this paper, where the size of the membership set is evaluated with a probabilistic confidence.

Theorem 2. Consider the system (1). Assume that the regressor $\phi_{i}$ is deterministic, periodic, and PE. In addition, $v_{i}$ and $\eta_{i}$ are independent random variables, and their bounds are tight. Then, the size of the membership set can be evaluated with a probabilistic confidence as

$$
\operatorname{Prob}\left\{\operatorname{dia} \Omega_{1}^{N}(\epsilon, \delta) \leq \frac{2 \sqrt{n}}{\alpha}(\rho+\mu \beta)\right\} \geq c_{p}>0
$$

where $c_{p}$ is a positive constant satisfying

$$
c_{p} \leq\left\{1-2\left(1-p_{v}(\rho) p_{\eta}(\mu)\right)^{\ell}\right\}^{n} .
$$

Proof: We first fix $i_{p}$ and consider the corresponding subsequence. For any $\hat{\theta}$, we have the identity

$$
\phi_{i_{p}}^{\mathrm{T}}(\theta-\hat{\theta})=y_{i_{p}+k n}-\phi_{i_{p}}^{\mathrm{T}} \hat{\theta}-\left(v_{i_{p}+k n}+\phi_{i_{p}}^{\mathrm{T}} \eta_{i_{p}+k n}\right)
$$

by rewriting (1). For $\hat{\theta}$ in the membership set, we see

$$
-\left(\epsilon+\delta\left\|\phi_{i_{p}}\right\|_{2}\right) \leq y_{i_{p}+k n}-\dot{\phi}_{i_{p}}^{\mathrm{T}} \hat{\theta} \leq \epsilon+\delta\left\|\phi_{i_{p}}\right\|_{2} .
$$

Thus, if there exists $k_{u}$ such that

$$
\begin{array}{r}
\epsilon-\rho+(\delta-\mu)\left\|\phi_{i_{p}}\right\|_{2} \leq v_{i_{p}+k_{u} n}+\phi_{i_{p}}^{\mathrm{T}} \eta_{i_{p}+k_{u} n} \\
\leq \epsilon+\delta\left\|\phi_{i_{p}}\right\|_{2}
\end{array}
$$

then we have

$$
\phi_{i_{p}}^{\mathrm{T}}(\theta-\hat{\theta}) \leq \rho+\mu\left\|\phi_{i_{p}}\right\|_{2} .
$$

In the same way, if there exists $k_{l}$ such that

$$
\begin{aligned}
-\epsilon-\delta\left\|\phi_{i_{p}}\right\|_{2} & \leq v_{i_{p}+k_{l} n}+\phi_{i_{p}}^{\mathrm{T}} \eta_{i_{p}+k_{l} n} \\
& \leq-(\epsilon-\rho)-(\delta-\mu)\left\|\phi_{i_{p}}\right\|_{2}
\end{aligned}
$$

then we also have the inequality

$$
-\left(\rho+\mu\left\|\phi_{i_{p}}\right\|_{2}\right) \leq \phi_{i_{p}}^{\mathrm{T}}(\theta-\hat{\theta}) .
$$

Hence, when such $k_{u}$ and $k_{l}$ both exist, it turns out that

$$
\left|\phi_{i_{p}}^{\mathrm{T}}(\theta-\hat{\theta})\right| \leq \rho+\mu\left\|\phi_{i_{p}}\right\|_{2}
$$

for any $\hat{\theta} \in \Omega_{1}^{N}(\epsilon, \delta)$. This implies

$$
\left|\phi_{i_{p}}^{\mathrm{T}}\left(\hat{\theta}_{1}-\hat{\theta}_{2}\right)\right| \leq 2(\rho+\mu \beta)
$$

for any $\hat{\theta}_{1}, \hat{\theta}_{2} \in \Omega_{1}^{N}(\epsilon, \delta)$, where $\left\|\phi_{i_{p}}\right\|_{2} \leq \beta$ follows (10). Therefore, we can see that

$$
\begin{aligned}
& \operatorname{Prob}\left\{\left|\phi_{i_{p}}^{\mathrm{T}}\left(\hat{\theta}_{1}-\hat{\theta}_{2}\right)\right| \leq 2(\rho+\mu \beta), \forall \hat{\theta}_{1}, \hat{\theta}_{2} \in \Omega_{1}^{\ell n}(\epsilon, \delta)\right\} \\
& \geq 1-2\left(1-p_{v}(\rho) p_{\eta}(\mu)\right)^{\ell}
\end{aligned}
$$

holds for this fixed $i_{p}$. This is because the probability that $v_{i_{p}+k n}+\phi_{i_{p}}^{\mathrm{T}} \eta_{i_{p}+k n}$ does not fall in (13) (or (14)) during $k=0,1, \ldots, \ell-1$ is $\left(1-p_{v}(\rho) p_{\eta}(\mu)\right)^{\ell}$ from Lemma 1 . Now, if (15) holds for all $i_{p}=1,2, \ldots, n$, we have

$$
\left\|\Phi^{n}\left(\hat{\theta}_{1}-\hat{\theta}_{2}\right)\right\|_{2} \leq 2 \sqrt{n}(\rho+\mu \beta)
$$

and thus we obtain

$$
\begin{aligned}
\left\|\hat{\theta}_{1}-\hat{\theta}_{2}\right\|_{2} & \leq\left\|\left\{\left(\Phi^{n}\right)^{\mathrm{T}} \Phi^{n}\right\}^{-1}\left(\Phi^{\dot{n}}\right)^{\mathrm{T}}\right\|_{i 2}\left\|\Phi^{n}\left(\hat{\theta}_{1}-\hat{\theta}_{2}\right)\right\|_{2} \\
& \leq \frac{2 \sqrt{n}}{\alpha}(\rho+\mu \beta)
\end{aligned}
$$

where $\left\|\left\{\left(\Phi^{n}\right)^{T} \Phi^{n}\right\}^{-1}\left(\Phi^{n}\right)^{\mathrm{T}}\right\|_{i 2} \leq 1 / \alpha$ follows (10). Here, to ensure (15) for all $i_{p}$, (16) should hold for all $i_{p}$, $i_{p}=1,2, \ldots, n$. We therefore conclude the proof.

Note that Theorem 2 says that

$$
\operatorname{Prob}\left\{\operatorname{dia} \Omega_{1}^{N}(\epsilon, \delta) \leq \frac{2 \sqrt{n}}{\alpha}(\rho+\mu \beta)\right\} \rightarrow 1
$$

for all $\rho$ and $\mu$ when we select

$$
c_{p}=\left\{1-2\left(1-p_{v}(\rho) p_{\eta}(\mu)\right)^{\ell}\right\}^{n} .
$$

Since $\rho$ and $\mu$ can be chosen arbitrarily, it turns out that, with probability one,

$$
\operatorname{dia} \Omega_{1}^{N}(\epsilon, \delta) \rightarrow 0 \text { as } \ell \rightarrow \infty
$$

Thus we conclude that the tightness implies convergence property of the membership set.

Although Theorem 2 gives a probabilistic upper bound of the diameter of the membership set, it also gives a deterministic upper bound if $c_{p}=1$, that is, $p_{v}(\rho)=$ $p_{\eta}(\mu)=1$. This selection is possible when we set $\rho=2 \epsilon$; $\mu=2 \delta$. Then, we obtain

$$
\operatorname{dia} \Omega_{1}^{N}(\epsilon, \delta) \leq \frac{4 \sqrt{n}}{\alpha}(\epsilon+\delta \beta)
$$

Comparing the above with (6) in Theorem 1, we see that the deterministic bound derived from Theorem 2 is twice as conservative as that of Theorem 1 .

Now, let us consider the case that $c_{p}$ is the probabilistic confidence that we want to ensure and $2 \sqrt{n}(\rho+\mu \beta) / \alpha$ is the bound of the diameter of the membership set that we want to specify. Note that $\rho$ and $\mu$ determine the bound. Thus, it would be preferable that we car calculate the necessary number of repetition $\ell$ which satisfies 
Table 1 Relation between confidence and tightness

\begin{tabular}{lrrrrr}
\hline$\hat{\mu}$ & & 0.05 & 0.01 & 0.005 & 0.001 \\
\hline$\ell$ & & 608 & 6772 & 19143 & 213917 \\
$c_{p}(\%)$ & 90.11 & 90.08 & 90.09 & 90.08 \\
$c_{m}(\%)$ & 100 & 100 & 100 & 100 \\
\hline
\end{tabular}

(11) for given $c_{p}, \rho$, and $\mu$. This can be done as follows. Suppose that the distributions of the disturbance and the parameter uncertainty are known. Then, the probability of $p_{v}(\rho) p_{\eta}(\mu)$ can be computed. Solving the inequality. (12), we have

$$
\ell \geq \frac{\ln \left(\frac{1-\left(c_{p}\right)^{1 / n}}{2}\right)}{\ln \left(1-p_{v}(\rho) p_{\eta}(\mu)\right)}
$$

This gives the necessary number of repetition $\ell$ explicitly.

\section{Numerical Example}

Let us consider the case that no additive disturbance exists for simplicity, that is,

$$
\begin{aligned}
& y_{i}=\phi_{i}^{\mathrm{T}}\left(\theta+\eta_{i}\right), \\
& \theta=\left[\begin{array}{l}
1 \\
1
\end{array}\right], \quad \phi_{i}=\left[\begin{array}{c}
u_{i-1} \\
u_{i}
\end{array}\right], \quad \eta_{i}=\left[\begin{array}{l}
\eta_{1 i} \\
\eta_{2 i}
\end{array}\right] .
\end{aligned}
$$

The parameter uncertainty $\eta_{i}$ satisfies $\left\|\eta_{i}\right\|_{2} \leq 0.1$ and is assumed to be an independent and uniformly distributed random variable. In this case, the probability on the tightness is calculated as

$$
p_{\eta}(\mu)=\frac{1}{\pi}\left(\cos ^{-1}(1-\hat{\mu})-(1-\hat{\mu}) \sqrt{2 \hat{\mu}-\hat{\mu}^{2}}\right)
$$

where $\hat{\mu} \doteq \mu / \delta$ is the index of tightness we choose later. We used the following sequence of the regressor

$$
\phi_{1}=\left[\begin{array}{l}
1 \\
2
\end{array}\right], \quad \phi_{2}=\left[\begin{array}{l}
2 \\
3
\end{array}\right], \quad \phi_{3}=\left[\begin{array}{l}
3 \\
1
\end{array}\right]
$$

which is periodic with period 3 and $\operatorname{PE}(\alpha=\sqrt{3}, \beta=5)$.

For several indices of tightness $\hat{\mu}$, we specified the confidence $c_{p}$ around $90 \%$ and calculated the necessary number of repetition $\ell$ satisfying (17). The results are shown in Table 1. Here $c_{m}$ is the measured confidence, each of which is the average of 10,000 trials. The significant figure. is four. Subsequently, choosing $5 \%, 10 \%$, and $15 \%$ of $\ell$ in Table 1, we also calculated the corresponding confidence $c_{m}$ by simulations. The results are shown in Table 2 .

Table 1 shows that $c_{p} \leq c_{m}$, thus we confirmed Theorem 2. However, there is a gap between $c_{p}$ and $c_{m}$. Note that a comment related to conservatism in Theorem 2 is stated in the second paragraph below the proof of the theorem. Table 2 shows how much conservative $c_{p}$ is from the view point of the number of repetition. For this example, we needed at least $10 \%$ and at most $15 \%$ of $\ell$ satisfying (17) to ensure that $c_{m}$ is around $90 \%$.
Table 2 Relation between confidence and the number of repetition

\begin{tabular}{lrrrrr}
\hline$\hat{\mu}$ & 0.05 & 0.01 & 0.005 & 0.001 \\
\hline$\ell(5 \%)$ & 31 & 339 & 958 & 10696 \\
$c_{m}(\%)$ & 62.87 & 63.17 & 63.33 & 63.05 \\
\hline$\ell(10 \%)$ & 61 & 678 & 1915 & 21392 \\
$c_{m}(\%)$ & 89.70 & 89.92 & 89.48 & 89.52 \\
\hline$\ell(15 \%)$ & 91 & 1016 & 2872 & 32088 \\
$c_{m}(\%)$ & 98.28 & 97.98 & 98.00 & 98.11 \\
\hline
\end{tabular}

\section{Concluding Remarks}

In this paper, we have investigated convergence properties of the membership set in a deterministic and a stochastic settings. In particular, we have shown a quantitative relationship between the diameter of the membership set and the number of samples. We have also derived the necessary number of samples which meets the specification for the diameter with a specified probabilistic confidence.

\section{References}

1) G. Belforte, B. Bona and V. Cerone: Parameter Estimation Algorithms for a Set-Membership Description of Uncertainty, Automatica, 26-5, 887/898 (1990)

2) E. Walter and H. Piet-Lahanier: Estimation of Parameter Bounds from Bounded-Error Data - A Survey, Mathematics and Computers in Simulation, 32, 449/468 (1990)

3) M. Milanese and A. Vicino: Optimal Estimation Theory for Dynamic Systems with Set Membership Uncertainty An Overview, Automatica, 27-6, 997/1009 (1991)

4) Special Issue on Bounded-Error Estimation, International Journal of Adaptive Control and Signal Processing, 8-1, (1994)

5) Special Issue on Bounded-Error Estimation, International Journal of Adaptive Control and Signal Processing, 9-1, (1995)

6) S. M. Veres and J. P. Norton: Structure Selection for Bounded Parameter Models - Consistency Condition and Selection Criterion, IEEE Transactions on Automatic Control, 36-4, 474/481 (1991)

7) E.-W. Bai, R. Tempo and H. Cho: Membership Set Estimators - Size,Optimal Input, Complexity and Relations with Least Squares, IEEE Transactions on Circuits and Systems, Part I, 42-5, 266/277 (1995)

8) E.-W. Bai, H. Cho and R. Tempo: Convergence Properties of the Membership Set, Automatica, 34-10, 1245/1249 (1998)

9) W. Kitamura and Y. Fujisaki: Probabilistic Approach to Set Membership Identification - The Size and Convergence Properties of the Membership Set, Proceedings of SICE 2nd Annual Conference on Control Systems, Makuhari, Japan, 25/28 (2002) (in Japanese)

10) M. Vidyasagar: A Theory of Learning and Generalization - With Applications to Neural Networks and Control Systems, Springer-Verlag, London (1997)

11) E. Weyer, R. C. Williamson and I. M. Y. Mareels: Finite Sample Properties of Linear Model Identification, IEEE Transactions on Automatic Control, 44-7, 1370/1383 (1999)

12) Y. Oishi: Learning-Theoretic System Identification for Ro- 
bust Control, Transactions of the Institute of Electronics, Information and Communication Engineers, J83-A-6, $651 / 660$ (2000) (in Japanese)

Appendix A. The case $\alpha=\beta$ and $n_{0}=m$

Let us define

$$
\Phi^{N}=\left[\begin{array}{llll}
\alpha I & \alpha I & \cdots & \alpha I
\end{array}\right]^{\mathrm{T}} .
$$

The PE condition with $\alpha=\beta$ and $n_{0}=m$. obviously holds for this $\Phi^{N}$. Furthermore, we see

$$
\left(\Phi^{N}\right)^{\mathrm{T}} \Phi^{N}=\beta^{2} \ell I
$$

and

$$
\left\|\phi_{i}\right\|_{2}=\beta
$$

for all $i$. Thus, from the characterization (4) of the membership set, there exist $\theta_{1}, \theta_{2} \in \Omega_{1}^{N}(\epsilon, \delta)$ such that

$$
y-\Phi^{N} \theta_{1}=-(\epsilon+\delta \beta) e, \quad y-\Phi^{N} \theta_{2}=(\epsilon+\delta \beta) e
$$

where

$$
e=\left[\begin{array}{llll}
1 & 1 & \cdots & 1
\end{array}\right]^{\mathrm{T}} .
$$

Then we have

$$
\Phi^{N}\left(\theta_{1}-\theta_{2}\right)=2(\epsilon+\delta \beta) e .
$$

We therefore see that

$$
\begin{aligned}
& \left\|\theta_{1}-\theta_{2}\right\|_{2}^{2} \\
& =\left(\theta_{1}-\theta_{2}\right)^{\mathrm{T}}\left\{\left(\Phi^{N}\right)^{\mathrm{T}} \Phi^{N}\right\}^{-1}\left(\Phi^{N}\right)^{\mathrm{T}} \Phi^{N}\left(\theta_{1}-\theta_{2}\right) \\
& =\frac{1}{\beta^{2} \ell}\left\|\Phi^{N}\left(\theta_{1}-\theta_{2}\right)\right\|_{2}^{2} \\
& =\frac{4 N}{\beta^{2} \ell}(\epsilon+\delta \beta)^{2}
\end{aligned}
$$

that is,

$$
\left\|\theta_{1}-\theta_{2}\right\|_{2}=\frac{2 \sqrt{m}}{\beta}(\epsilon+\delta \beta)
$$

This means

$$
\operatorname{dia} \Omega_{1}^{N}(\epsilon, \delta) \geq\left\|\theta_{1}-\theta_{2}\right\|_{2}=\frac{2 \sqrt{m}}{\beta}(\epsilon+\delta \beta)
$$

and with Theorem 1, we conclude

$$
\operatorname{dia} \Omega_{1}^{N}(\epsilon, \delta)=\frac{2 \sqrt{m}}{\beta}(\epsilon+\delta \beta) .
$$

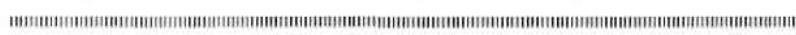

\section{Wataru Kitamura (Student Member)}

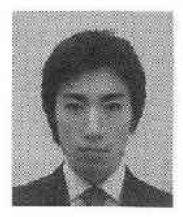

He received the B. E. and M. E. degrees from Kobe University, Japan in 2000 and 2002 respectively. $\mathrm{He}$ is currently a doctoral course student of Graduate school of Science and Technology, Kobe University. He is a member of ISCIE. His research interests include system identification and robust control theory.

\section{Yasumasa Fujisaki (Member)}

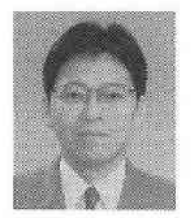

He received his B.S., M.S., and Ph.D. degrees from Kobe University, Japan, in 1986, 1988, and 1994, respectively. From 1988 to 1991, he was a Research Member of the Electronics Research Laboratory, Kobe Steel, Ltd., Japan. In 1991, he joined the faculty of Kobe University, where he is currently an Associate Professor of Department of Computer and Systems Engineering. He has held a visiting academic appointment at IRITI-CNR, Politecnico di Torino, Italy, from 2000 to 2001. His research interests include probabilistic methods for analysis and design of control systems. He is a member of ISCIE, ISIJ, and IEEE.

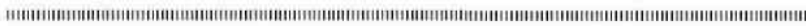

